\title{
On the Origin of the Amorphous Component in Polymer Single Crystals and the Nature of the Fold Surface
}

\author{
John D. Hoffman and G. Thomas Davis \\ Institute for Materials Research, National Bureau of Standards, Washington, D.C. 20234
}

(June 30, 1975)

\begin{abstract}
A model for the surface of folded-chain polymer single crystals is presented in which the "amorphous" phase is composed of polymer molecules physically adsorbed on surface sites of a fairly regularly folded surface. The evidence for the presence of an amorphous phase in polymer single crystals is reviewed briefly as well as the evidence for regular folding and adjacent reentry. The proposed model would allow simultaneous acceptance of the evidence for both an amorphous layer and a surface composed of regularly folded molecules; such evidence was heretofore contradictory. Experimental evidence for such a model is discussed and some predictions are made concerning the properties of such an adsorbed layer.
\end{abstract}

Key words: Adsorbed layer; amorphous component; fold surface; polymer crystals; regular folds.

The objective of this note is to present a possible resolution to the problem of "regular" folds versus an "amorphous" layer as a model for the surface of a chain-folded polymer single crystal. There is a considerable body of experimental evidence (e.g., density and heat of fusion data) that points to the existence of an "amorphous" layer that is in some way associated with the fold surface in single crystals. This has been widely interpreted as leading inexorably to the conclusion that most of the folds are either long and loose, or that nonadjacent reentry with intervening loops occurs on a large scale, or both (fig. 1A). On the other hand, there also exists an equally credible body of experimental evidence, based mainly on crystallographic, spectroscopic, and morphological observations, that suggests that certain polymer single crystals exhibit rather regular folding with mostly adjacent reentry (fig. 1B). From this it is frequently concluded that there is little place for the amorphous layer suggested by the density and heat of fusion data. Thus, acceptance of the aforementioned conclusions leads to a paradox, since "regular" folding and the presence of an "amorphous" component have been hitherto regarded as mutually exclusive or contradictory concepts. In the present work we shall suggest that the amorphous layer can in fact exist in most (but perhaps not all) cases, and that when it occurs that it is to a considerable extent the result of polymer molecules that are physically adsorbed on a fairly regularly folded surface (fig. 1C). The adsorbed layer is expected to resemble a two-dimensional random coil (or a highly flattened three-dimensional one) with "loops" or traverses between the points of attachment on the fold surface, and may be detachable under appropriate circumstances. If this suggestion is correct, the paradox is essentially resolved, since then neither the evidence for an amorphous layer nor that for rather regular folding with mostly adjacent reentry need be rejected.

Lauritzen and Hoffman were originally led to the suggestion of an adsorbed layer on polymer crystals by analysis of growth rate and lamellar thickness data [1]. ${ }^{1}$ Parameters resulting from the best fit of the data could be interpreted in terms of a model where a three-dimensional random coil in the subcooled liquid or solution state was adsorbed on the lateral surface as a quasi two-dimensional coil, the segments of which then migrated across that surface to the site of crystallization. This led to the subsequent prediction that a broadly similar adsorption effect probably took place on the fold surface [2].

Before proceeding with the main discussion, we briefly outline the experimental evidence leading to the apparently contradictory conclusions noted above regarding the fold surface. In the interest of brevity, we concentrate on polyethylene, and even this case is not treated exhaustively.

The density of polyethylene single crystals formed from solution has been studied extensively. Despite some early data showing near-perfect single crystal densities [3], the main body of results [4-6] in the literature leave little doubt on two points. One is that the density is well below that of a "perfect" crystal predicted from unit cell dimensions $\left(\rho_{25^{\circ} \mathrm{C}}=1.00 \mathrm{~g} / \mathrm{cm}^{3}\right)$

\footnotetext{
${ }^{1}$ Figures in brackets indicate the literature references at the end of this paper.
} 
A

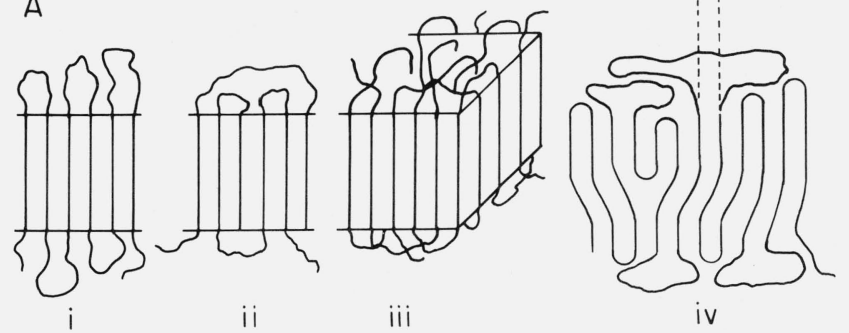

B
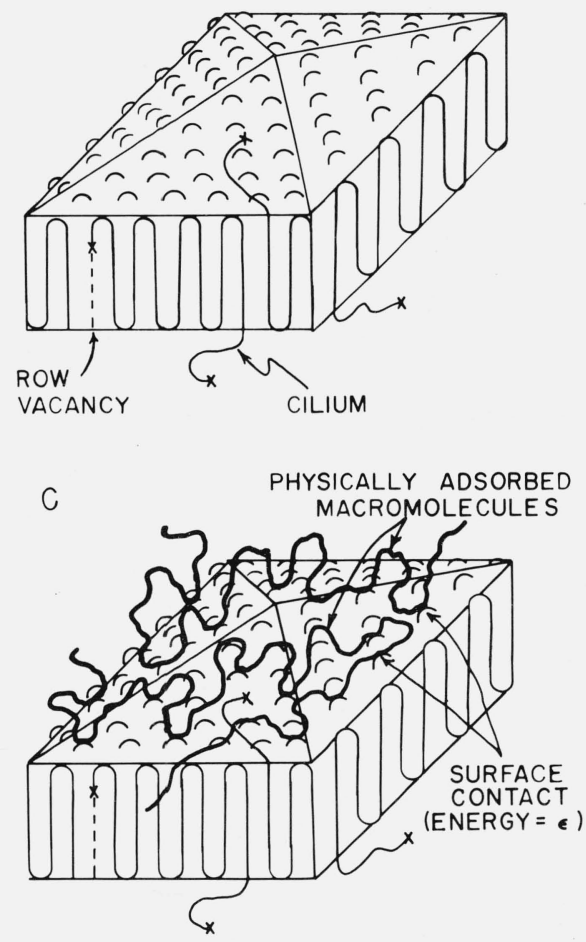

FIGURE 1. Models for polymer single crystals (schematic).

A. Models with loose loops where amorphous phase is part of the fold zone: (i) adjacent reentry with loose loops in growth plane (see ref. [11]), (ii) nonadjacent reentry with loops in growth plane (see ref. [13]), (iii) nonadjacent reentry, commonly known as the "switch board" model (see ref [14]), (iv) mostly adjacent reentry with some loose folds and some "buried" folds (see ref. [12]).

B. Model with regular folds and adjacent reentry forming a smooth or slightly rough fold surface with no amorphous phase.

C. Proposed model with mostly adjacent reentry and smooth or slightly rough fold surface and same crystal defects as in B, but with an amorphous phase that consists mostly of polymer molecules that are physically adsorbed on sites on the fold surface.

and the other is that the density increases as the crystals become thicker [7-10]. Several models for a noncrystalline phase associated in some manner with the fold surface have been proposed to account for this density deficiency. Zachmann [11] has considered loose loops between adjacent crystal sequences whereas Keller et al. [12] have attributed it to a combination of loose loops and "buried" folds. A surface region composed of long cilia and loose loops resulting from nonadjacent reentry within the growth plane was suggested by Frank [13] and a more or less random reentry of molecules into the crystal has been advanced by Flory [14]. (The presence of a density defect, however, does not necessarily prove the existence of extensive nonadjacent reentry or a large number of loose loops.) We note that the density of the single crystals of a given overall thickness has been reported to depend upon the solvent from which the crystal was grown [9], e.g., crystals from $n$-hexadecane were consistently about $0.004 \mathrm{~g} / \mathrm{cm}^{3}$ more dense than those of equal thickness grown from tetralin and decalin. This implies that the nature of the amorphous surface layer is dependent on the solvent. It has also been suggested that the density of the crystals may increase slightly with time when stored in the presence of solvent [4].

Studies of the heat of fusion of polyethylene single crystals have also been made which point to the conclusion that the observed heat of fusion for single crystals is always substantially below the theoretical value for a crystal of infinite thickness $\left(\Delta h_{f} \cong 293 \mathrm{~J} / \mathrm{g}\right)$ [15-17]. A small part of the reduced heat of fusion can be explained by the heat stored in the folds [2] (circa $15 \mathrm{~J} / \mathrm{g}$ ) but the deficiency is still so large as to clearly suggest the existence of a noncrystalline component that is in some way associated with the fold surface of the crystal. More recently, low angle x-ray intensity measurements on single crystal mats of polyethylene have been interpreted in terms of a two-phase model $[18,19]$. Also, the low angle x-ray spacing of single crystal mats has been shown to increase and then decreases reversibly upon the addition and subsequent removal of solvents [20], implying the existence of an amorphous zone capable of being swollen by solvent. A more detailed study [21] has shown that the magnitude of the increase in low-angle spacing depends upon the molecular weight of the polymer and the crystallization conditions, from which it may be inferred that the nature of the amorphous surface layer depends upon these variables. A slightly rough fold surface could account for part of the density deficiency but it cannot account for all of the other observations.

The above is sufficient to establish the necessity of considering the existence of an amorphous layer of some type on single crystals of polyethylene. Studies on other polymers are sufficient in scope to establish the phenomenon as a quite general one. For the sake of brevity, we have omitted evidence based on relaxation measurements (NMR, dielectric, and mechanical).

Consider now the evidence for fairly "regular" folding in polymer single crystals. By "regular" we mean folding with mostly adjacent reentry and without a substantial fraction of long loop-like folds. Certainly some "mistakes" such as occasional nonadjacent reentry in the growth plane must be allowed, as well as some defects such as row vacancies and cilia (figs. $1 \mathrm{~B}$ and $1 \mathrm{C}$ ). Also, a slight roughness of the fold surface including a few "buried folds" may be permitted, but nothing to the extent shown in figure lA is intended under the definition of "fairly regular."

Geil [22] has summarized the crystallographic and morphological evidence of many workers, notably Bassett, Frank, and Keller, and Reneker and Geil, and Niegisch and Swan, for essentially regular folding in polyethylene single crystals of various types. Al- 
though many cases are discussed, the evidence is epitomized by his discussion of hollow pyramidal single crystals of polyethylene. The best way to explain the existence of these well-known sectorized single crystals is the staggered regular packing of chain folds by analogy with a terra cotta roof. It would seem practically impossible to explain the details of the crystal structure and morphology of such crystals with any of the models shown in figure $1 \mathrm{~A}$. There is nothing inconsistent with the explanations summarized by Geil in case the rather regular fold surface were subject to an adsorbed layer of polymer molecules: all that would be added would be the amorphous layer suggested by other measurements.

Further evidence for rather regular chain folding with predominantly adjacent reentry lies in the nature of the fracture in different sectors of the same crystals [23] and the slight difference in crystallographic spacing in adjacent sectors of the same crystal [24]. Clear evidence for mostly adjacent reentry in polyethylene single crystals has been given by Bank and Krimm [25] based on an analysis of the IR spectra of mixed crystals of deuterated and hydrogen bearing chains. Finally, we mention the work of Holland and Lindenmeyer [26], who found dislocation networks between polyethylene single crystals, and the more extensive work of Sadler and Keller [27] who showed the phenomenon to be specific for molecular weights below 3000. Dislocation networks provide clear evidence for crystallographic contact between layers and hence regularity of the folds - in this case it is clear that no substantial amorphous layer intervenes between the crystals. (Subsequently, we shall suggest possible reasons for the virtual absence of an amorphous layer in material of sufficiently low molecular weight.) In any case, it is clearly not admissible to ignore the sum of the evidence for fairly regular folding in polyethylene crystals formed from dilute solution.

Accordingly, if the experimental evidence for an amorphous layer and for fairly regular folding is simultaneously accepted, one is led to the model shown in figure $1 \mathrm{C}$ which depicts a crystal with a fairly regular fold surface and an independent surface layer composed of physically adsorbed polymer. We may now ask if there is any evidence for such a model.

Direct experimental evidence for the existence of a thin mechanically detachable layer on single crystals of several polymers has been found by Jones (nee Breedon) and Geil [28, 29]. (See preceding article in this journal where details are given for the case of poly(oxymethylene).) From this work there is good reason to believe that single crystals of a number of polymers of normal molecular weight possess a mechanically detachable layer on their fold surfaces. Accordingly, it is believed that the model shown in figure $1 \mathrm{C}$ is a reasonable representation of the situation. The adsorbed molecules on the fold surface provide the amorphous component suggested by heat of fusion and density measurements among other results, and simultaneously provide the material that strips off the fold surface during fracture. The underlying fold surface itself could be fairly regular (including mostly adjacent reentry) in keeping with the requirements of crystallographic, morphologic, and spectroscopic evidence. The proposed representation is a true "two-phase" model with a quite abrupt phase boundary between the two phases (as suggested by Fischer et al. [18] and as shown experimentally by Strobl and Müller [19]).

We turn now to certain practical and theoretical considerations relating to adsorption of polymer molecules on surfaces. Considerable insight may be obtained by sketching out what is known about polymer adsorption on foreign substrates, since little revision is needed to adapt this information to the case of adsorption of molecules on chain folds consisting of the same monomer units.

Theoretical calculations by various workers [30-33] show that an isolated polymer molecule will form something that roughly resembles a two-dimensional or an appreciably flattened three-dimensional random coil on a foreign substrate. Each single surface contact has an energy taken to be $\epsilon$. Traverses or "loops" intervene between runs of adsorbed segments. Defining $\theta$ as the fraction of polymer segments adsorbed for each molecule, statistical mechanical calculations have been made giving $\theta$ as a function of $\epsilon / k T$ for various chain lengths. In general, the results show an increasing $\theta$ with increasing values of $\epsilon / k T$, as shown schematically in figure 2A. Although somewhat dependent on the specifics of the model chosen [33], it is characteristic of such calculations to show a low value of $\theta$ for $|\epsilon| k T \mid<<1$, and a relatively high value of $\theta$ for values of $|\epsilon| k T \mid \geqslant 1$. In the case of a monomer unit attaching itself at some appropriate point on a chain fold, where this point represents a surface site, values of $\epsilon$ that are roughly comparable to $k T$ do not seem unreasonable. Even a low $\theta$, arising from a low value of $\epsilon / k T$, can involve a considerable amount of amorphous polymeric material being associated with the fold surface; in this case most of the mass of the amorphous material will be in loops between occupied surface sites. The total amount of the material on the surface will depend on the molecular weight and the crystallization conditions, including the solvent from which the crystal was formed, so some variation in the average thickness of the adsorbed layer is to be expected. In broad aspect, this probably underlies the differences noted earlier in the amorphous fraction detected in polyethylene single crystals crystallized from different solvents [9] and the dependence of the amount of swelling on molecular weight and temperature of crystallization $[20,21]$.

Experiments on the rate of adsorption and desorption of polymer molecules on foreign substrates $[34,35]$ show two important facts that are relevant to the suggested model shown in figure 1C. First, the rate of adsorption is rapid compared to the rate of desorption. In the case of polystyrene on a chromium surface the rate of desorption is extremely slow: in some cases 80 percent of the original adsorbed polymer still remains after 3 weeks. The basic reason for the low rate of desorption is that, even when $\epsilon$ is rather small, all the points of contact have to be removed in the period of time required for diffusion away from the 


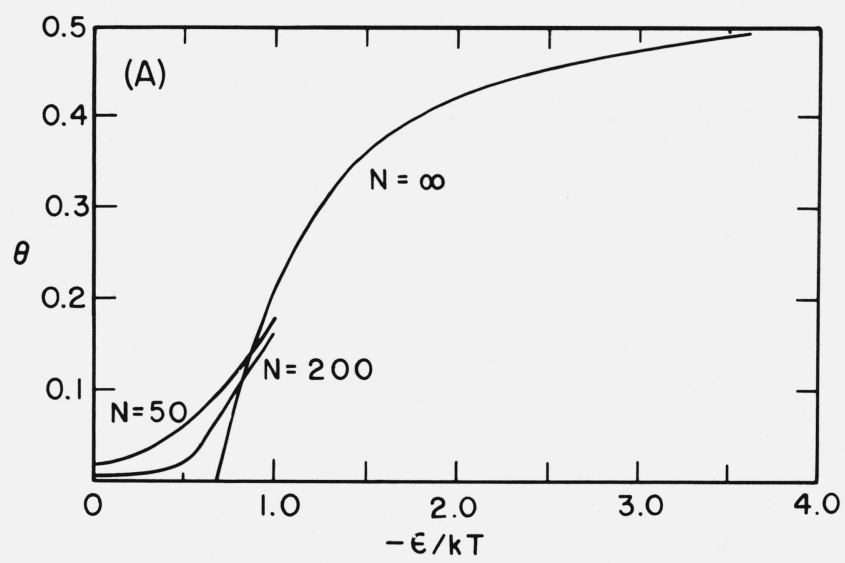

(B)

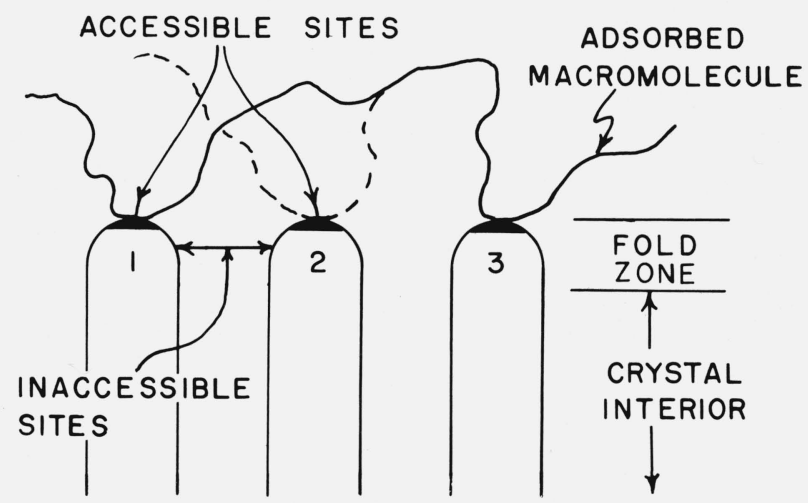

FIGURE 2. Physical adsorption of macromolecules on surfaces (schematic).

A. Fraction of chain segments $\theta$ adsorbed at the surface as a function of $-\epsilon / k T$. The quantity $\epsilon$ is the energy of attachment per chain segment and $N$ is the number of segments in the chain (adapted from ref. [31]).

B. Model showing possible origin of reduced mobility of molecules adsorbed on fold surface.

surface, which is a statistically improbable event [34]. As applied to the present situation, these results imply that the adsorbed layer on the chain fold surface will very likely be highly persistent for material of moderate and high molecular weight. This leads us to the second point, which is that the rates of adsorption and desorption are a function of molecular weight [35]. When expressed as mass per unit area, the lower molecular weight polymer adsorbs more slowly and desorbs more rapidly than does the higher molecular weight. Accordingly the adsorbed surface layer on a fold surface may be tenuous in specimens of low molecular weight. (In such cases cilia are expected to be more numerous, and contribute to the "amorphous" character of the crystal.) In moderate and high molecular weight material, the adsorbed layer will have more segments in contact with the surface and desorb much more slowly. This may explain the fact that dislocation networks, which require intimate contact between two crystals over a considerable area, are found in low molecular weight polyethylene single crystals, but not in ones of moderate to high molecular weight [27].
It has been noted earlier [2] that the adsorbed layers on the lateral surface are apparently mobile, and move across that surface to the site of crystallization. We must now raise the question as to why the material postulated to exist on the fold surface does not share the same fate as that adsorbed on the lateral surface, namely, rapid crystallization. We speculate that a reduced surface mobility of the polymer molecules on the fold surface may be explained as follows. Referring to figure $2 \mathrm{~B}$, it is seen that it is reasonable to propose that a considerable distance intervenes between "allowed" sites for attachment of the adsorbed polymer molecule; movement which results in the molecule going from site 1 to site 2 requires total desorption because of the intervening "inaccessible" sites resulting from spatial considerations. Because the sites are much closer together on the lateral surface, the barriers to be surmounted for mobility could be considerably smaller on this surface. (The mobility on the lateral surface in bulk polymers varies as exp $\left[-U^{*} / R\right.$ $\left.\left(T-T_{\infty}\right)\right]$ where $U^{*} \cong 1500 \mathrm{cal}(6275 \mathrm{~J})$ and $T_{\infty} \cong T_{g}-$ $\left.30^{\circ}\right)[2]$. We note that our basic concept here would eventually permit migration to a lateral surface and some crystallization of the layer adsorbed on the fold surface. However, it is reasonable to expect this to be very slow (except in low molecular weight material) because of the postulated reduced mobility of the molecules adsorbed on the fold surface, and the large distances over which transport of molecules would have to take place to reach the lateral surface where crystallization would take place. Such a slow migration followed by crystallization may account for the increase of density found in aged preparations of single crystals [4]. It should be mentioned that the presence of long cilia [36] could also account for many of the observations described above but one would not expect such a layer to be easily detached as in the fracture experiments nor removable by prolonged storage in solvent.

Though it is necessarily highly speculative, some comments on how the adsorbed amorphous surface layer might be reduced or even removed are in order. It is already clear from the work of Jones and Geil that portions of the layer can be removed in fracture experiments, but the area deprived of the amorphous layer is small in these cases, and we therefore concentrate on techniques that might reduce or remove the layer on a larger scale. It has already been remarked that certain solvents yield higher density crystals, implying the presence of less amorphous material. Crystallization from extremely dilute solution for long periods of time might also yield crystals with less adsorbed material. Beginning with such crystals, it might be possible by long extraction in proper solvents below the dissolution temperature of the crystal to dissolve the amorphous layer and remove it or eventually crystallize it by prolonged storage. It is conceivable that the presence of appropriate liquids may tend to render the amorphous layer on the fold surface more mobile, and allow it to crystallize. Progress in removing the amorphous layer could be detected by density or heat of fusion measurements, or in extreme cases, by looking for dislocation 
networks. Removal of the adsorbed layer by chemical attack is problematical, since one must expect the strained bonds in the folds to also be ruptured at a rather early time. However, the molecular weight of the adsorbed layer might be reduced by brief chemical attack in a manner sufficient to allow its removal by other means.

Consider now the properties to be expected of the adsorbed layer on the fold surface. It need not be of constant amount and density in all preparations but may depend on molecular weight, crystallization temperature, solvent used and time of storage. In the presence of liquids of good solvent power, the layer may be solvated and expanded. In the presence of poorer solvents, it should be less expanded and rather flattened onto the fold surface. In the dried or contracted state, the layer is expected to have a density. not far from that of the corresponding subcooled liquid or glassy state. In relaxational studies, the "loops" between the point of contact should exhibit the mobility and chain motions typical of short-range motions at low temperatures in the glassy state (i.e., the $\gamma_{a}$ transition) [37], but it is impossible to state at this juncture whether or not a true glass transition (i.e., $a \beta$ relaxation) with a typical $T_{g}$ should appear; we would anticipate that any $T_{g}$ that did appear would be weak and have abnormal characteristics. Few, if any, single crystal preparations show a $\beta$ relaxation in dynamic mechanical or dielectric measurements.

A slow increase of density and heat of fusion on prolonged storage in the presence of solvent may be found, resulting from a low but finite surface mobility with subsequent crystallization. In general, the hypothesis of an adsorbed surface layer on a fairly regular fold surface offers a vehicle for alternative explanations of a number of phenomena observed for polymer single crystals.

Although this paper deals primarily with the possibility of an adsorbed layer on polymer single crystals, a number of the considerations may apply to polymers crystallized from the melt. This possibility is discussed in a recent review [2], where it is brought out that an adsorbed layer may account for a considerable portion of the amorphous content $\alpha=1-\chi$ in highly crystalline specimens $(\chi \cong 0.85$ to $\chi=0.90)$. Amorphous material in concentrations higher than $\alpha=0.1$ to 0.15 or so may be ascribed at least in part to the presence of interlamellar entanglements that prevent crystallization on a large scale. DiMarzio and Rubin [32] have carried out calculations for the adsorption of molecules between two parallel plates. A long molecule frequently adsorbs on both plates, producing an amorphous link between them. The analogous situation may occur in polymers crystallized from the melt, leading to an increased amorphous content.

\section{References}

[1] Lauritzen, J. I., Jr., and Hoffman, J. D., J. Appl. Phys. 44, 4340 (1973).

[2] Hoffman, J. D., Davis, G. T., and Lauritzen, J. I., Jr., in Treatise on Solid State Chemistry, N. B. Hannay, Ed., (Plenum Press, New York, Vol. 3, Chapter 6, 1976).

[3] Kawai, T., and Keller, A., Philos. Mag. 8, 1203 (1963).

[4] Martin, G. M., and Passaglia, E., J. Res. Nat. Bur. Stand. (U.S.), 70A (Phys. and Chem.) No. 3, 221-224 (May-June 1966).

[5] Blackadder, D. A., and Lewell, P. A., Polymer 9, 249 (1968).

[6] Sharma, R. K., and Mandelkern, L., Macromolecules 2, 266 (1969).

[7] Fischer, E. W., and Lorenz, R., Kolloid Z. Z. Polym. 189, 97 (1963).

[8] Kitamaru, R., and Mandelkern, L., J. Polym. Sci. A-2, B, 2079 (1970).

r [9] Nakajima, A., Hayashi, S., Korenaga, T., and Sumida, T., Kolloid Z. Z. Polym. 222, 124 (1967).

[10] Davis, G. T., Weeks, J. J., Martin, G. M., and Eby, R. K., J. Appl. Phys. 45, 4175 (1974).

[11] Zachmann, H. G., Z. Naturforschg. 19A, 1937 (1964).

[12] Keller, A., Martuscelli, E., Priest, D. J., and Udagawa, Y., J. Polym. Sci., A-2, 9, 1807 (1971).

[13] Frank, F. C., referred to by A. Keller, Kolloid Z. Z. Polym. 231, $386(1969)$.

[14] Flory, P. J., J. Am. Chem. Soc. 84, 2857 (1962).

[15] Fischer, E. W., and Hinrichsen, G., Polymer 7, 195 (1966); Kolloid Z. Z. Polym. 213, 93 (1966).

[16] Hendus, H., and Illers, K. H., Kunststoffe 57, 193 (1967).

[17] Mandelkern, L., Allou, A. L., Jr., and Gopalan, M., J. Phys. Chem. 72, 309 (1968).

[18] Fischer, E. W., Goddar, H., and Schmidt, G. F., J. Polym. Sci. B5, 619 (1967).

[19] Strobl, G. R., and Müller, N., J. Polym. Sci. Polym. Phys. Ed., 11, 1219 (1973).

[20] Udagawa, Y., and Keller, A., J. Polym. Sci. A-2, 9, 437 (1971).

[21] Ergïz, E., and Mandelkern, L., J. Polym. Sci. B 1 0, 631 (1972).

[22] Geil, P. H., Polymer Single Crystals, Interscience, New York, pp. 125-139 (1963).

[23] Lindenmeyer, P. H., J. Polym. Sci. C1, 5 (1963).

[24] Bassett, D. C., Philos. Mag. 12, 907 (1965).

[25] Bank, M. I., and Krimm, S., J. Polym. Sci. A-2, 7, 1785 (1969).

[26] Holland, V. F., and Lindenmeyer, P. H., J. Appl. Phys. 36, 3049 (1965).

[27] Sadler, D. M., and Keller, A., Kolloid Z. Z. Polym. 239, 641 (1970) and 242, 1081 (1970).

[28] Jones, J. B., and Geil, P. H., J. Res. Nat. Bur. Stand. (U.S.), 79A (Phys. and Chem.), No. 5, 609-611 (Sept.-Oct. 1975).

[29] Breedon, J. E., Crack Formation in Polymer Single Crystals, Masters Thesis, Case Western Reserve Univ. (1975).

[30] Silberberg, A., J. Chem. Phys. 46, 1105 (1967).

[31] DiMarzio, E. A., and McCrackin, F. L., J. Chem. Phys. 43, 539 (1965).

[32] DiMarzio, E. A., and Rubin, R. J., J. Chem. Phys. 55, 4318 (1971).

[33] Rubin, R. J., J. Chem. Phys. 43, 2392 (1965).

[34] Stromberg, R. R., Grant, W. H., and Passaglia, E., J. Res. Nat. Bur. Stand. (U.S.), 68A (Phys. and Chem.), No. 4, 391-399, (July-Aug. 1975).

[35] Grant, W. H., Smith, L. E., and Stromberg, R. R., to be published in Discuss. Faraday Soc. (1975).

[36] Sanchez, I. C., and DiMarzio, E. A., J. Chem. Phys. 55, 893 (1971).

[37] Hoffman, J. D., Williams, G., and Passaglia, E., J. Polym. Sci. C14, 173 (1966).

(Paper 79A5-862) 